\title{
Une représentation du risque à l'intersection de l'aléa et de la vulnérabilité: cartographies des inondations Iyonnaises
}

\author{
F. Renard and D. Soto \\ Université Jean Moulin Lyon 3, UMR 5600 Environnement Ville Société CNRS, Lyon, France \\ Correspondence to: F. Renard (florent.renard@univ-lyon3.fr)
}

Received: 3 January 2015 - Revised: 4 July 2015 - Accepted: 16 July 2015 - Published: 10 November 2015

\begin{abstract}
Résumé. Essential to risk studies, vulnerability analysis of the assets of an area is often not suitable for practical application. It is usually more an assets census than a real vulnerability assessment, facing a given hazard. Its intersection with the hazards zoning poses also many methodological, cartographic and operational problems. This work starts with the key concepts of risk, thanks to a review of French and international literature study. Then, it provides a procedure which allows precise knowledge of the local vulnerability, available to all types of hazards and applicable to all areas. Finally, in order to get an overall perspective of risk, assets vulnerabilities are crossed with the hazards areas, in accordance to the spatial distribution of both components and using innovative geomatics operations. As part of this study, this methodology will be applied to the rivers flooding and overflowing runoff of the Greater Lyon.
\end{abstract}

\section{Introduction: le Grand Lyon, un territoire exposé aux inondations}

La communauté urbaine de Lyon (Grand Lyon), au nord du couloir Rhodanien, dans le sud-est français, concentre 1,3 millions d'habitants (Fig. 1). L'inondation est le risque le plus fréquent et le plus coûteux sur ce territoire (Renard, 2010), tout comme dans la majorité du reste du monde (UNISDR, 2011; Jongman et al., 2014). Depuis les années 2000, l'inondation est l'aléa naturel le plus fréquent et le plus coûteux, avec 16,2 milliards de dollars de dégâts. Elle est en outre la deuxième catastrophe faisant le plus de victimes (1054), derrière les événements liés aux températures extrêmes ${ }^{1}$. Elle peut être provoquée par des débordements des deux principaux cours d'eau du Grand Lyon (Rhône et Saône), à cinétique lente mais de grande envergure. Des crues éclairs à caractère torrentiel (flash floods - Gaume et al., 2009; Llasat et al., 2010; Marchi et al., 2010; Tarolli et al., 2012) sont également possibles sur tout l'ouest lyonnais, parcouru par de multiples ruisseaux affluents du Rhône, caractérisés par leurs fortes pentes (Fig. 1). L'inondation par

\footnotetext{
${ }^{1}$ Source: http://www.emdat.be
}

ruissellement pluvial urbain pose en outre beaucoup de problèmes, dans le centre du Grand Lyon mais aussi dans l'est lyonnais, en plaine, dépourvue de réseau hydrographique naturel et fortement imperméabilisée. Enfin, des inondations sont possibles dans le centre urbain après de fortes pluies, liées à la remontée de la nappe phréatique. Pratiquement tout le Grand Lyon est donc susceptible d'être impacté par une submersion des enjeux de son territoire.

Si les aléas (phénomènes physiques de nature, d'intensité et d'occurrence donnée) associés à ces risques sur le territoire lyonnais ont fait l'objet de très nombreuses études spécifiques (Kouyi et al., 2011; Becouze-Lareure, 2012; Lepioufle et al., 2012; Braud et al., 2013), les études portant sur l'étude de la vulnérabilité territoriale du Grand Lyon sont rares et ont en commun d'être relativement critiquables et perfectibles (notamment Agence d'urbanisme pour le développement de l'agglomération lyonnaise, 2005; Rufat, 2007; Renard et Chapon, 2010, sur ce même territoire). En effet, la gestion du risque, historiquement fondée sur le contrôle et la limitation des aléas, s'est tournée plus tardivement vers une tentative de réduction de la vulnérabilité des enjeux. En outre, la prise en compte simultanée de l'exposition aux aléas et de la vulnérabilité territoriale n'a pas donné de résultats 

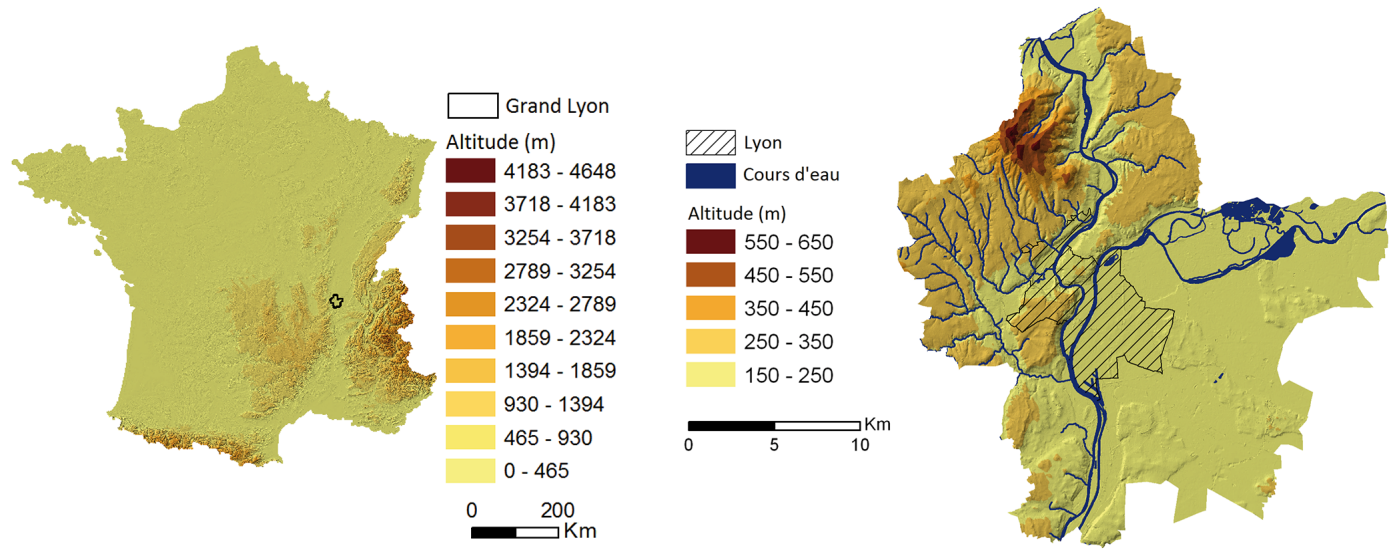

Figure 1. Localisation du Grand Lyon en France (gauche), et détails de sa topographie et de son hydrologie (droite).

probants (comme dans Granger, 2009; Caradot et al., 2011, par exemple), en raison de lacunes qui seront expliquées dans la troisième partie du texte. Cette étude propose ainsi une méthodologie de cartographie des risques d'inondation centrée sur l'exposition de la vulnérabilité des enjeux aux aléas considérés, qui est appliquée au Grand Lyon, mais transposable à n'importe quel territoire. Ces termes sont discutés en détail dans la première partie de cet article.

\section{Rappel essentiel sur les éléments constituant le risque}

\subsection{La notion de risque: une notion polysémique}

Le risque, notion polysémique, partagée par de nombreuses disciplines, a de nombreuses définitions (Füssel et Klein, 2006; O’Brien et al., 2007). "Une quantité de définitions ont été proposées par les géographes et les autres disciplines depuis des années, avec des lacunes persistantes, des redondances, des confusions, quand ce n'est pas des contradictions entre les disciplines" (Moser, 2010). "En fonction de l'approche prise en compte, sa définition varie considérablement, l'accent étant tantôt mis sur les aspects fonctionnels et techniques, tantôt sur les dimensions sociales et psychologiques" (Thomi, 2010). Il est le plus souvent perçu comme le produit des interactions entre une société et son environnement sur un espace donné (Kron, 2002; UNISDR, 2011; IPCC, 2014; Poussin et al., 2012, par exemple). "Inhérent au système territorial" (Meschinet de Richemond et Reghezza, 2010), il est "profondément enraciné dans le territoire" (November, 2002). Il s'agit d'une "notion composite, [...] considérée comme la concrétisation potentielle de phénomènes plus ou moins prévisibles et ayant des répercussions le plus souvent négatives dans l'espace et le temps" (Demoraes et D'Ercole, 2009). Il est ainsi le "résultat de la rencontre entre un élément perturbateur de nature aléatoire et un élément vulnérable" (Chocat, 1997), "la probabilité de perte affectant des objets (les enjeux) soumis à un événement dom- mageable (l'aléa)" (Veyret et Reghezza, 2006), ou encore “ quelque chose de potentiel, qui ne s'est pas encore produit, mais dont on pressent qu'il se transformera en événement néfaste pour les individus ou une collectivité dans un ou des espaces donnés" (November, 2002). Ainsi, "l'approche traditionnelle du risque naturel repose sur la distinction entre deux composantes du risque, l'aléa, qui désigne le processus physique source de danger, et la vulnérabilité, qui renvoie à la propension de l'enjeu à subir un endommagement" (Meschinet de Richemond et Reghezza, 2010). Pour Bourrelier (2006), "le couple aléa/vulnérabilité est la clé de gestion de tous les risques". "Il est désormais admis que le risque naît de la conjonction d'une menace (l'aléa) et d'enjeux plus ou moins vulnérables" (Léone, 2007), selon leur exposition physique, c'est-à-dire "la nature et le degré auquel un système est exposé à un aléa" (Füssel et Klein, 2006), ou encore la combinaison de la fréquence des aléas et des populations exposées (Peduzzi et al., 2009).

\subsection{L'aléa, longtemps uniquement pris en compte}

"Longtemps naturaliste, la géographie française s'est d'abord consacrée à l' analyse de l'aléa avant de s'ancrer dans les sciences sociales" (Veyret et Reghezza, 2006). Ainsi, la gestion du risque a longtemps été focalisée sur la maîtrise de l'aléa, et depuis quelques dizaines d'années, la connaissance de l'aléa a fait "des progrès considérables: les phénomènes sont identifiés, mesurés, modélisés, recadrés historiquement, actualisés, cartographiés” (Bourrelier, 2006). Ainsi, “jusqu'à la fin des années 1980, les cartes dites de risque "naturel" ne comportaient le plus souvent qu'un fond topographique sur lequel étaient superposées les zones exposées à tel ou tel type de phénomènes (inondation, glissement de terrain, couloir d'avalanche, etc.)" (Demoraes et D'Ercole, 2009), c'està-dire un zonage de l'aléa.

Dans son sens général, "l'aléa exprime la mesure d'une probabilité de situation, d'un événement ou d'une cause quelconque. Il suggère dans le domaine des risques naturels 
la représentation du danger en prenant un rôle actif, matérialisé par ses caractéristiques spatio-temporelles et son intensité" (Haziza, 2007). Il est ainsi perçu comme un événement menaçant pouvant occasionner des dommages, caractérisé par une intensité au cours d'une période donnée et sur une étendue donnée, et par une probabilité d'occurrence. Griot et Ayral (2001) le voient comme "une possibilité d'apparition d'un phénomène résultant de facteurs ou de processus qui échappent au moins en partie à l'homme". Pour Propeck-Zimmermann et al. (2009), "l'aléa est entendu comme la probabilité d'occurrence d'un événement pouvant entraîner des effets dommageables dans une aire donnée". Cependant, "l'évolution actuelle des pratiques et des réglementations conduit à attacher de plus en plus d'importance à la vulnérabilité des milieux exposés à la perturbation" (Chocat, 1997). En outre, d'après (Gilbert, 2006), "l'aléa ne serait que l'occasion de l'expression des vulnérabilités".

\subsection{La vulnérabilité: une prise de conscience récente et nécessaire}

Contrairement à l'aléa, le terme de vulnérabilité ne fait son apparition que très tardivement dans les études (Ruin et Lutoff, 2004; Veyret et Laganier, 2013; Koks et al., 2015). Il se substitue à "des appellations aussi diverses que sensibilité, susceptibilité, fragilité" (Veyret et Reghezza, 2006). Haziza (2007) la voit comme la "réponse territoriale à l'aléa". Elle est perçue par Griot et Ayral (2001) comme "un indice de fragilité des éléments à risque par rapport à l'occurrence d'un événement", de même que par Veyret et Reghezza (2006) comme "le degré d'endommagement dû à l'exposition des enjeux". Pour Blaikie et al. (1994), la vulnérabilité est le degré de perte d'enjeux sur lesquels un aléa d'une sévérité donnée pourrait se produire. Ces définitions rejoignent celle de l'Intergovernmental Panel on Climate Change (IPCC, 2014). Pour d'Ercole et Pigeon (1999), elle est "la propension d'une société donnée à subir des dommages, en cas de manifestation d'un phénomène naturel ou anthropique". Cette définition est très proche de celle de Propeck-Zimmermann et al. (2009) pour lesquels "la vulnérabilité exprime la propension des enjeux à subir des dommages". Dans cette étude, la vulnérabilité correspond à la proposition de Gleyze et Reghezza (2007) comme " la propension d'un enjeu humain, matériel, environnemental, etc. à subir un dommage". Sera donc considéré comme vulnérable tout enjeu dont l'intégrité se trouverait impactée par la manifestation d'un aléa sur celui-ci.

La relation entre un aléa et la vulnérabilité des enjeux crée ainsi la notion de risque. Cependant, la relation existante entre les deux termes de ce binôme est ambiguë. En effet, pour Chocat (1997), "la vulnérabilité est totalement indépendante de l'aléa", mais elle se manifeste cependant lors de l'occurrence de ce dernier. Cette notion est partagée par la plupart des auteurs, dont notamment Veyret et Reghezza (2006) pour lesquelles "la vulnérabilité est [...] une propriété intrinsèque d'une société, d'un groupe social, d'un individu, un facteur interne du risque, quasi indépendante de l'aléa au sens où elle préexiste à ce dernier; mais qui est cependant révélée par lui".

Du nombre élevé de définitions présentes dans la littérature et propres à chaque auteur, il ressort du concept de vulnérabilité son caractère plurivoque (Shi et Zeng, 2013; Lummen et Yamada, 2014; Marzo et al., 2015). Ainsi, "la caractérisation de la vulnérabilité s'avère ainsi être très souvent un exercice extrêmement délicat" (Haziza, 2007). Cependant, une gestion efficace des risques sur un territoire nécessite d'évaluer de façon intégrée les conjonctions des différents paramètres d'aléas mais aussi de vulnérabilités (Reghezza, 2009).

\subsection{Les enjeux du territoire vulnérables ?}

Dans la littérature relative à la notion de risque, le terme "enjeu" est aussi très fréquemment rencontré, devenu même quasiment indissociable de la notion de vulnérabilité. Les enjeux constituent l'ensemble des personnes et des biens directement menacés par l'aléa, qui sont susceptibles de subir des préjudices ou des dommages (Griot et Ayral, 2001). Pour Propeck-Zimmermann et al. (2009), "l'enjeu définit tout ce qui peut subir des dommages ou dysfonctionnements et fait référence à la composition et au fonctionnement global du territoire (population, bâti, milieu naturel, transport ... )". Enfin, pour Demoraes et D’Ercole, (2009), ils constituent "par définition des éléments essentiels au fonctionnement et au développement d'un territoire. Leur endommagement, défaillance ou perte seraient en conséquence particulièrement préjudiciable pour le territoire en question". Ces enjeux peuvent être affectés par divers aléas, et l'évaluation de leur vulnérabilité est l'objectif de la prochaine partie.

\section{Une méthode hiérarchique multicritère d'aide à la décision pour l'analyse de la vulnérabilité territoriale}

\subsection{La diversité des évaluations de la vulnérabilité urbaine et leurs limites}

En France, la gestion des risques naturels repose essentiellement sur la prévention par l'urbanisme et les réglementations. Le plan de prévention des risques naturels (PPRN), instauré par la loi du 2 février 1995, établi par commune ou par bassin de risque et élaboré par l'état, en est l'instrument, en faisant connaître les zones à risque et en règlementant l'urbanisation locale. Il réglemente l'utilisation des sols en fonction des risques naturels présents, et sont approuvés par le préfet, après enquête publique et consultation des communes, puis annexés au plan local d'urbanisme (PLU). Le document final comprend un rapport sur les aléas avec des cartographies détaillées et leurs impacts sur les personnes et les biens. D'après l'Office Fédéral de l'Environnement (OFEV) 
suisse $^{2}$, le PPRN français et sa partie sur les aléas est proche de la carte des dangers suisse, qui contient des informations sur les types de dangers et leur importance, exprimées par les différents degrés de danger (rouge, bleu, jaune, hachuré jaune et blanc), et sur l'étendue des zones menacées, de l'échelle 1 : 2000 à l'échelle 1 : 10000 . Cependant, il n'existe pas réellement d'étude opérationnelle de la vulnérabilité dans les réglementations françaises et suisses. Pourtant, plusieurs auteurs ont proposé des tentatives d'évaluation de la vulnérabilité, notamment sur Lyon, dont la méthodologie, les objectifs et l'applicabilité varient. La plupart de ces études se limitent généralement à la prise en compte de la densité résidentielle et les études plus complètes sont très rares (Fedeski et Gwilliam, 2007; Kaźmierczak et Cavan, 2011; Fuchs et al., 2012; Kappes et al., 2012). En effet, d'après Griot (2007), “ l'analyse d'une vingtaine d'études de vulnérabilité montre qu'elles ne tiennent souvent pas compte de l'ensemble des éléments vulnérables". Un constat similaire est fait ici, quel que soit le territoire d'étude, localement comme à l'international. En effet, les enjeux matériels et les enjeux environnementaux sont généralement exclus de ces évaluations, et se résument bien souvent à l'analyse du nombre de résidents par communes ou, dans de rares cas, par découpages administratifs plus fins. En outre, l'applicabilité opérationnelle de ces études est souvent limitée, contrainte par des échelles d'étude inadaptées, par le type des enjeux utilisés et par la méthode employée. Ainsi, cette étude, fondée sur le territoire du Grand Lyon, adopte une démarche plus complète, qui vise à englober tous les enjeux présents. Si les aléas associés aux risques sur ce territoire ont fait l'objet de nombreux travaux, les études portant la vulnérabilité territoriale sont rares et présentent des faiblesses méthodologiques (notamment Combe, 2007; Rufat, 2007; Renard et Chapon, 2010). Il convient toutefois de remarquer que la gestion du risque a longtemps été focalisée sur la maîtrise de l'aléa, quel que soit son lieu d'application ou sa nature (Veyret et Reghezza, 2006), et n'est pas une caractéristique du site d'étude ou de ses gestionnaires.

Cette évaluation de la vulnérabilité territoriale du Grand Lyon doit être objective et les conclusions doivent être partagées par le plus grand nombre afin d'aboutir à un consensus en vue d'une application opérationnelle. Pour cela, des méthodes d'aide à la décision reposant sur des méthodes hiérarchiques multicritères et le jugement d'experts sont utilisées.

\subsection{Choix d'une méthode d'aide à la décision pour l'évaluation de la vulnérabilité des enjeux du territoire}

Les méthodes d'aide à la décision interviennent pour faciliter les problèmes de choix de différentes alternatives, de décision ou d'évaluation dans des situations complexes, où plusieurs critères qualitatifs et quantitatifs entrent en jeu. Elles permettent d'en agréger plusieurs avec l'objectif de sélec- tionner une ou plusieurs actions, options ou solutions. Ici, l'objectif est de prioriser les différents enjeux en fonction de leur vulnérabilité face à l'aléa. Il est donc nécessaire de recourir à une procédure de classement, afin de les pondérer. Plusieurs méthodes d'aide à la décision existent, avec chacune leurs avantages et leurs faiblesses (tableau 1). L'utilisation d'une méthode plutôt qu'une autre est définie par des paramètres principaux tels que les objectifs, la simplicité d'utilisation, la finesse de l'évaluation, la flexibilité, le temps nécessaire à leur mise en œuvre et le retour d'expérience. Après une évaluation de ces différentes techniques (dans Renard, 2010), notre choix s'est tourné vers la comparaison par paire, et plus précisément l'Analytic Hierarchy Process (AHP - Saaty, 1980). Elle permet d'ordonner les alternatives sur la base soit d'un critère unique, soit de différents critères appréhendés dans leur pluralité.

L'AHP est une théorie de mesure de critères d'une situation donnée, fondée sur la dérivation de priorités d'importance relative à partir de comparaisons par paires d'alternatives homogènes partageant un attribut commun (Saaty, 1994; Kendrick et Saaty, 2007). Cette méthode emprunte à l'approche systémique (se focalisant sur le fonctionnement de l'ensemble) et à l'approche déductive (interrelation des parties) pour structurer une situation complexe en différents éléments pouvant interagir les uns avec les autres, afin de les quantifier pour leur attribuer des valeurs relatives à leurs impacts sur le système global (Griot, 2003). Cette quantification des valeurs des différents éléments repose sur l'expérience et le jugement d'experts, pour, à terme, obtenir des pondérations des différentes composantes: ici, les fonctions de vulnérabilité.

Cette méthode d'analyse hiérarchique multicritère repose ainsi sur cinq étapes principales qui vont être mises en œuvre dans la partie suivante, et qui sera complété par une cartographie du risque (Fig. 2):

- description détaillée du système et des enjeux pour caractériser, synthétiser et décomposer la situation complexe

- construction des hiérarchies afin d'organiser les enjeux pour répondre à la problématique, ceci de manière structurée et la plus complète possible

- entretiens semi-dirigés, où les experts concernés se prononcent par comparaison binaire sur les enjeux leurs semblant posséder une vulnérabilité à la submersion plus élevée que les autres

- validation de la cohérence des réponses des experts et calcul de la pondération des enjeux

- agrégation des réponses fournissant les fonctions de vulnérabilité

${ }^{2}$ http://www.bafu.admin.ch/ 
Tableau 1. Méthodes de pondération utilisables lors de l'analyse multicritères (adapté de Cherqui, 2005).

\begin{tabular}{l|l}
\hline Avantages & Inconvénients \\
\hline \multicolumn{1}{c}{ Comparaison deux à deux des critères. } \\
\hline $\begin{array}{l}\text { Simple d'utilisation, la cohérence de l'ensemble des } \\
\text { comparaisons est vérifiée, et permet d'appréhender la } \\
\text { complexité du monde réel }\end{array}$ & $\begin{array}{l}\text { Le temps de comparaison augmente plus rapidement } \\
\text { que le nombre de critères }\end{array}$ \\
\hline
\end{tabular}

Technique du jeu de cartes

Distribution d'un "jeu de cartes" sur lesquelles sont inscrits les noms de critères ainsi que des cartes blanches. Le décideur classe les cartes; il a la possibilité de mettre des cartes ex aequo et de glisser des cartes blanches entre les critères.

\begin{tabular}{l|l} 
Simplicité d'utilisation & $\begin{array}{l}\text { Difficulté d'appréhender la complexité globale de la réalité. } \\
\text { La pondération obtenue ne reflétera pas systématiquement } \\
\text { les différences d'importance entre critères }\end{array}$
\end{tabular}

Méthode d'attribution de scores

Répartition d'une somme de points sur l'ensemble des critères

\begin{tabular}{|c|c|}
\hline $\begin{array}{l}\text { L'attribution d'un poids plus important à un critère réduit } \\
\text { l'importance relative d'un autre élément }\end{array}$ & Mêmes inconvénients que la technique du jeu de cartes \\
\hline \multicolumn{2}{|c|}{$\begin{array}{l}\text { Arbre des pondérations } \\
\text { Lorsqu'il est possible de décomposer les critères sous forme d'une arborescence, alors à partir de la } \\
\text { pondération de chaque embranchement, la valeur de pondération de chaque critère correspond au } \\
\text { produit des coefficients des sous-branches et des branches dont il est issu. }\end{array}$} \\
\hline Le nombre d'éléments à comparer est limité & Réalisation de l'arbre ardue \\
\hline \multicolumn{2}{|c|}{$\begin{array}{l}\text { Analyse de jugement } \\
\text { Evaluation de l'importance d'alternatives distinctes réelles ou fictives sur des échelles diverses. }\end{array}$} \\
\hline $\begin{array}{l}\text { Les pondérations sont fonction des comportements } \\
\text { révélés des acteurs et non pas de leurs préférences } \\
\text { officielles, et la méthode permet la prise en compte } \\
\text { indirecte de paramètres }\end{array}$ & $\begin{array}{l}\text { Pour obtenir une signification statistique, un nombre suffisant } \\
\text { d'alternatives doit être étudié. La méthode conduit à surestimer } \\
\text { parfois la capacité cognitive des décideurs lorsque le nombre } \\
\text { d'alternatives est important. Chronophage }\end{array}$ \\
\hline
\end{tabular}
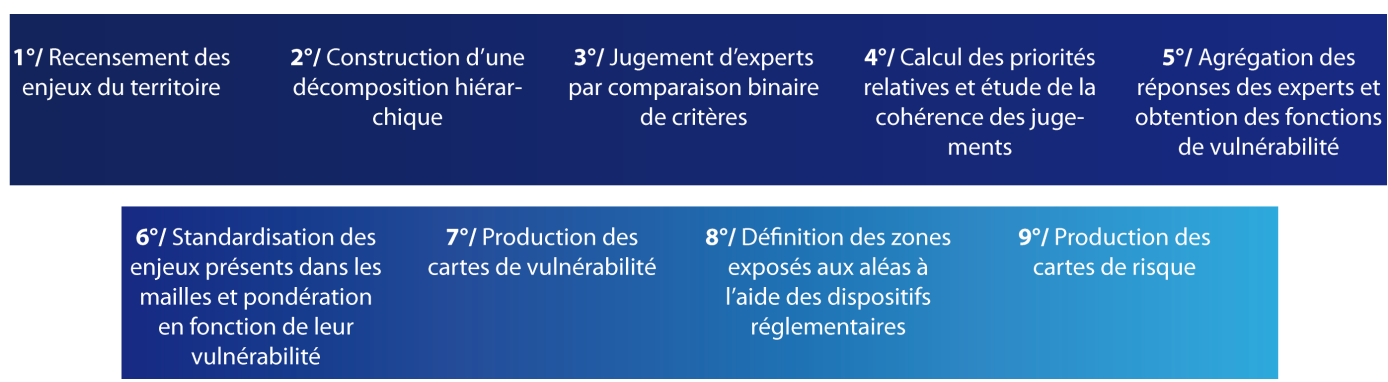

Figure 2. Schéma général de la méthodologie.

\section{Application géomatique de la représentation du risque au Grand Lyon}

\subsection{Hiérarchisation structurée du système}

Une décomposition hiérarchique est créée, afin d'avoir une description globale de la situation (Tixier et al., 2006). Une hiérarchie spécifique à ce territoire est ainsi construite, fon- dée sur une typologie combinant les trois principales classes d'enjeux (humains, environnementaux et matériels) et leurs facteurs de vulnérabilité (Fig. 3).

Les enjeux humains, matériels et environnementaux sont chacun de six grands types principaux, détaillés en Fig. 3. Ils peuvent être dotés, le cas échéant, de facteurs de sensibilité (indiqués en Fig. 3). Les facteurs de sensibilité, comme le proposent Léone (2007), Propeck-Zimmermann et al. (2009) 
ou Veyret et Laganier (2013), sont des caractéristiques intrinsèques de l'enjeu considéré qui affectent la vulnérabilité de ce dernier. En effet, pour les enjeux matériels, "les caractéristiques des constructions modulent la vulnérabilité de l'habitat" (Combe, 2007), et pour les enjeux environnementaux, les facteurs de sensibilité peuvent prendre en compte l'état des champs ainsi que l'avancement et le type des cultures. Les facteurs de sensibilité des enjeux humains permettent d'affiner le recours à la simple densité résidentielle. Cette densité est souvent considérée comme le facteur de vulnérabilité le plus évident (Blancher et al., 1996). Cependant, elle retranscrit dans le même temps la concentration des ressources pouvant permettre une réaction rapide et efficace aux situations d'urgence, bien que d'après les forces de l'ordre et les pompiers (Service Départemental d'Incendie et de Secours du Rhône), l'ensemble des communes du Grand Lyon soit accessible en moins de dix minutes (Rufat, 2007). Du fait de ces caractéristiques discutables, l'analyse de la population résidente doit être affinée par des facteurs de sensibilité, tout comme les autres types de population. En effet, pour un aléa similaire, les conséquences des catastrophes diffèrent d'une catégorie de personnes à une autre. D'après Dauphiné et Provitolo (2013), la population âgée de moins de dix ans et celle de plus de 75 ans sont les plus fragiles et les plus difficiles à évacuer en cas de nécessité. Il en est de même pour les personnes à mobilité réduite. En outre, les personnes en habitat de fortune sont plus fragiles et plus facilement exposées à toutes sortes d'aléa (Rufat, 2007) et le niveau d'étude a aussi été employé comme indicateur de la capacité à trouver des solutions en cas de catastrophes, capacité proportionnelle au degré de formation (Kreimer et al., 2003; Veyret et Laganier, 2013), nous amenant à prendre en compte la population âgée de plus de quinze ans sans diplômes.

La construction de cette structure hiérarchique s'est appuyée sur l'utilisation du recensement de 2012 de l'Institut National de la Statistiques et des Etudes Economiques (INSEE) à l'échelle de l'IRIS, pour les enjeux humains ${ }^{3}$. Les enjeux environnementaux et matériels sont renseignés grâce à la base de données du système d'information géographique (SIG) du Grand Lyon, à la base Georhonealpes (ex-Prodige) de la Direction Régionale de l'Environnement, de l'Aménagement et du Logement (DREAL), et au SAGE de l'Est Lyonnais. Cependant, les facteurs de sensibilité des enjeux matériels et environnementaux n'ont, pour le moment, été pris en compte, faute de temps.

Cette hiérarchisation des enjeux lyonnais a été construite en collaboration avec les acteurs locaux du Grand Lyon.

${ }^{3}$ L'IRIS (Ilots Regroupés pour l'Information Statistique) est un découpage du territoire en maille homogène, stable dans le temps, et constitue la brique de base en matière de diffusion de données infra-communales. Leur population se situe en général entre 1800 et 5000 habitants. Ils sont homogènes quant au type d'habitat et leurs limites s'appuient sur les grandes coupures du tissu urbain (voies principales, voies ferrées, cours d'eau, etc.)
Cependant, elle n'est en aucun cas définitive, et les enjeux comme les facteurs de sensibilité ont la possibilité d'être enrichis en fonction de son utilisation opérationnelle et de l'obtention de connaissances sur cette problématique. Cette capacité d'amélioration en continu constitue un des points forts de cette méthode et favorise ainsi son usage par les acteurs locaux.

\subsection{Le recours aux jugements d'experts pour le calcul des priorités}

L'évaluation de la vulnérabilité des enjeux repose sur le jugement d'une quarantaine d'experts locaux (ingénieurs de collectivités locales, de bureau d'études, ou d'universitaires) dérivé à l'aide d'un calcul matriciel. Les experts sont pour moitié des employés de collectivités locales (ingénieurs et techniciens de la fonction publique territoriale), de bureaux d'études (Véolia, Lyonnaise des eaux, Egis, etc.: $30 \%$ ), et d'universitaires $(20 \%)$, choisis pour leurs implications professionnelles dans l'aménagement du territoire, la planification urbaine, la gestion et la prévention des risques. Ils ont en outre validé la décomposition hiérarchique préalablement établie (cf. Sect. 4.1).

Cette quantification est fondée sur des comparaisons d'enjeux deux à deux, à l'aide d'une échelle spécifique basée sur les critères de Saaty (1980 - tableau 2). Cette grille d'évaluation possède l'avantage de mesurer des jugements subjectifs aussi bien que formellement quantitatifs, afin de mesurer le degré de priorité d'un des enjeux de la hiérarchie par rapport à un autre. Ainsi, chacun des enjeux est comparé à tous les autres enjeux du même ordre (et relativement à chacun des enjeux du niveau supérieur). Il est ainsi demandé à l'expert d'évaluer la vulnérabilité des enjeux de même nature qui se situent au même rang de la décomposition hiérarchique, pour une hauteur d'eau de $50 \mathrm{~cm}$ environ. Cette hauteur a été choisie en concertation avec les agents territoriaux de la métropole car elle correspond au contexte local (Lyon n'est ni en région méditerranéenne, ni en région cévenole, où des inondations beaucoup plus sévères sont fréquentes), et qu'il s'agit d'une hauteur moyenne minimale critique qui impacte les réseaux électriques domestiques. D'autres variables telles que la pollution des eaux, la vitesse ou la durée de l'inondation peuvent aussi jouer un rôle sur la gravité de l'impact, mais elles sont relativement difficiles à mesurer (Caradot et al., 2011). En outre, elles sont souvent fortement corrélées avec la hauteur de l'inondation et ainsi soit mises de côté, soit prises en compte en tant que paramètres secondaires uniquement (Caradot et al., 2011; Messner et Meyer, 2006; Penning-Rowsell et al., 2005).

Les matrices de comparaison renseignées à l'aide des jugements d'experts permettent de calculer la vulnérabilité de chacun des enjeux. En outre, un ratio de cohérence est calculé afin de valider la cohérence des réponses fournies. En effet, par exemple, si un expert se prononce pour un plus grand poids de l'enjeu A par rapport à l'enjeu B, et pour 


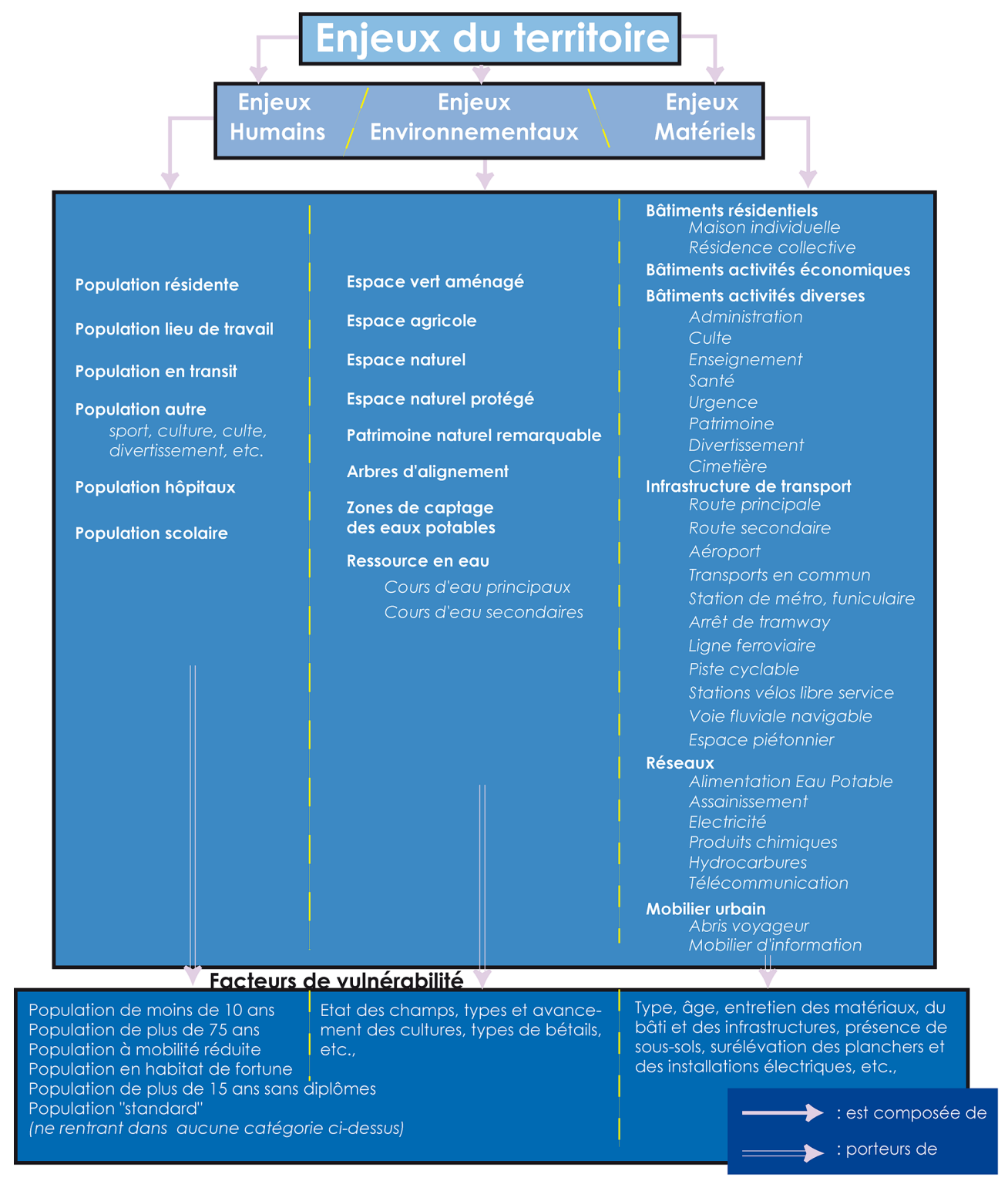

Figure 3. Décomposition hiérarchique généralisée du système spatial de la vulnérabilité lyonnaise.

un plus grand poids de l'enjeu $\mathrm{B}$ par rapport à l'enjeu $\mathrm{C}$, en toute cohérence il devra se prononcer pour un plus grand poids de l'enjeu A par rapport à l'enjeu C (notion de transitivité des jugements). La cohérence de ces réponses est estimée à l'aide d'un ratio issu de la valeur propre des matrices de comparaison et qui doit être inférieur à $10 \%$. Dans le cas contraire, les réponses inconsistantes sont éliminées. Ensuite, les réponses sont agrégées par une moyenne géométrique, telle que conseillée par l'auteur, pour reconstituer une matrice unique de comparaison (Tixier et al., 2006). Enfin, les vecteurs propres des matrices carrées de jugement ont été calculés pour fournir les fonctions qui déterminent les pondérations.

\subsection{Réflexion sur le cadre d'analyse: des enjeux de taille et de forme différentes}

L'AHP a ici pour but d'établir des pondérations de vulnérabilité entre les enjeux présents sur le territoire du Grand Lyon (et recensés dans la Fig. 3). Cependant, une fois ces priorités établies au travers de fonctions de vulnérabilité (équation donnant la proportion de chacun des enjeux en fonction de leur vulnérabilité, par niveau de la décomposition hiérarchique), et pour une utilisation opérationnelle, il convient de les concrétiser visuellement par un travail cartographique. Il est donc nécessaire de recourir à un SIG pour spatialiser, traiter, stocker et analyser les informations hétérogènes concernant les enjeux. Le SIG permet ainsi de cartographier la vulnérabilité des enjeux selon les différentes fonctions de vul- 
Tableau 2. Echelle de comparaison binaire utilisée pour évaluer la vulnérabilité territoriale (adaptée de (Saaty, 1980).

\begin{tabular}{ll}
\hline $\begin{array}{l}\text { Pondération } \\
\text { numérique }\end{array}$ & Pondération verbale \\
\hline 1 & $\begin{array}{l}\text { Vulnérabilité égale des deux éléments } \\
\text { Un élément est un peu plus vulnérable } \\
\text { que l'autre }\end{array}$ \\
5 & Un élément est plus vulnérable que l'autre \\
7 & Un élément est beaucoup plus vulnérable \\
& que l'autre \\
9 & Un élément est absolument plus vulnérable \\
& que l'autre \\
$2,4,6,8$ & Valeurs intermédiaires entre deux \\
$1 / 2,1 / 3$, & appréciations voisines \\
$1 / 4,1 / 5$, & Valeurs réciproques des appréciations \\
$1 / 6,1 / 7$, & \\
$1 / 8,1 / 9$ & \\
\hline
\end{tabular}

nérabilité, en permettant une interrogation quantitative, i.e. en fonction de la quantité de l'enjeu présent dans la maille, ou qualitative, i.e. en fonction de la vulnérabilité de l'enjeu à l'aléa.

Cependant, la représentation graphique des enjeux se présente sous différentes natures spatiales: surfaciques, linéaires, ou ponctuelles. Il se pose alors la question du secteur d'exploration des enjeux car ils doivent être standardisés pour leur combinaison. De précédentes études reposaient sur les limites communales, sur les Iris, ou sur des “zones d'occupation homogène ou dominante" (Combe, 2007). En raison de leur diversité de taille, ces secteurs d'étude ne permettaient pas de réaliser des comparaisons quantitatives d'une zone à une autre, si ce n'est d'une manière relative en divisant la superficie des enjeux présents dans chacune des zones par celle du territoire. Outre des superficies très hétérogènes, les diversités de forme des communes ou des Iris ne permettaient pas une comparaison d'une zone à l'autre et nuisaient à l'interprétation des résultats. Ainsi, afin de travailler sur les données brutes (et non relatives en fonction de l'aire) et faciliter la comparaison des zones et la compréhension des résultats en s'affranchissant des limites communales ou de celles de l'INSEE, nous avons opté pour une standardisation de taille et de forme des secteurs d'exploration. Un maillage présentant des secteurs de taille et de forme identiques est ainsi utilisé. Deux types de carroyage sont couramment employés: la maille carrée (quadrillage) ou la maille hexagonale (fréquemment appelée carroyage en "nid d'abeilles" (Kienberger et al., 2009). Nous avons choisi le quadrillage pour des raisons de simplicité de forme évidente. Ce carroyage de la zone d'étude nous semble ainsi beaucoup plus approprié et opérationnel que les échelles spatiales utilisées lors des précédentes études sur le Grand Lyon.
La taille des mailles doit être adaptée au niveau de précision des données, mais aussi à l'espace étudié en lui-même et à sa problématique propre. Un carroyage de 100 mètres de côté est retenu (Fig. 4) et la maille d'une superficie de $10000 \mathrm{~m}^{2}$ offre un bon compromis entre la taille du secteur étudié ( 515 kilomètres carrés ${ }^{2}$ ), et le nombre de mailles ainsi créées (89 804).

Un carroyage moins fin a été utilisé par Renard et Chapon (2010), avec des mailles de $500 \mathrm{~m}$ de côté. Bien que les résultats cartographiques aient permis de faire ressortir certaines zones vulnérables, le rendu imprécis rendait les résultats inexploitables par les acteurs locaux. L'utilisation d'un carroyage plus fin permet d'affiner quantitativement et qualitativement la structuration des enjeux, et ainsi limite l'uniformisation de l'information, car les enjeux ne se répartissent pas forcément toujours d'une manière homogène dans la maille.

Les enjeux sont pondérés, à l'aide des fonctions de priorités préalablement établies, selon leur nature, mais doivent aussi l'être en fonction de leur emprise sur le terrain dans chacune des mailles. Un facteur de quantification est défini comme une variable sans dimension, s'échelonnant de 0 à 1 . La valeur 0 indique une absence de l'enjeu dans la maille, et la valeur 1 signifie que la quantité de cet enjeu est la plus forte comparativement aux autres mailles. Ainsi, les enjeux surfaciques (ex: zones de captage des eaux), linéaires (ex: réseau d'eau potable) et ponctuels (ex: arbres d'alignement) sont convertis par SIG sous une forme maillée, en divisant respectivement leurs aires, longueurs ou somme d'éléments dans la maille, par l'aire maximale, la longueur maximale ou la somme d'élément maximale de l'enjeu considéré dans une des mailles du territoire. De cette manière, les quantités d'enjeux présents dans une maille sont standardisés, indépendamment de leur nature (surfacique, linéaire ou ponctuelle) et de leur unité de mesure $\left(\mathrm{m}^{2}, \mathrm{~m}\right.$ ou somme, respectivement). Les facteurs de quantification permettent donc d'obtenir une quantification normalisée pour chacun des enjeux (Fig. 4) et des représentations standardisées de la vulnérabilité des enjeux du Grand Lyon, et cela quelle que soit la nature des enjeux et le niveau de la décomposition hiérarchique considéré. Enfin, il a été choisi de travailler en mode vectoriel plutôt qu'en raster pour tous les avantages procurés par le premier (rapidité d'affichage, espace de stockage réduit, précision, informations complémentaires stockées en table attributaire, etc.).

\subsection{Une tentative de cartographie des risques combinant vulnérabilité territoriale et périmètres d'aléas}

Après avoir défini les différentes vulnérabilités territoriales, la dernière étape de cette étude correspond à la confrontation de ces dernières avec les zonages d'aléas, tels que définis par exemple dans les Plans Locaux d'Urbanisme (PLU) ou dans les Plans de Prévention des Risques d'inondation (PPRI). Ce 
Représentation des enjeux sous forme :

surfacique

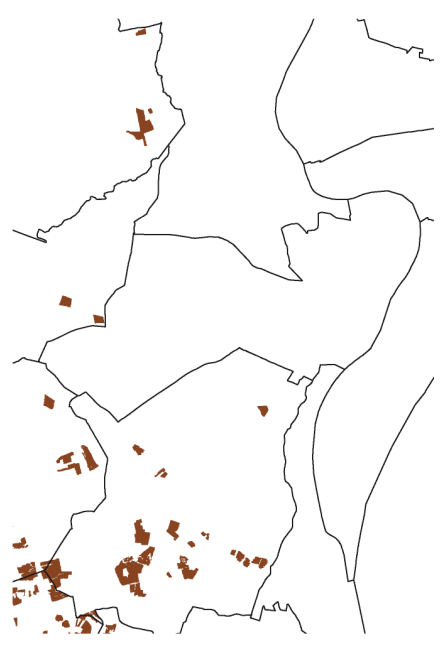

Espaces agricoles linéaire

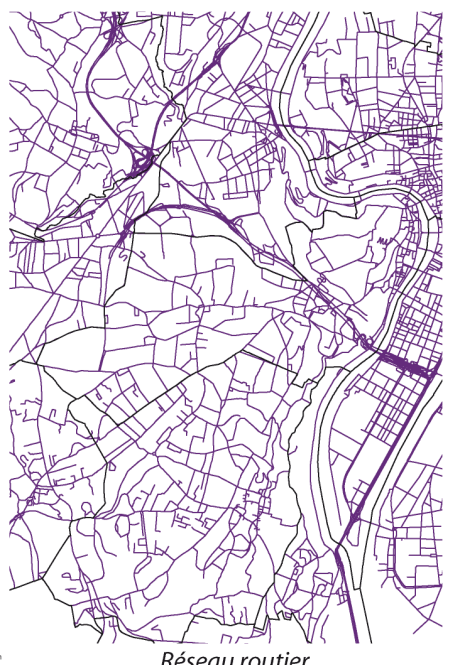

Réseau routier ponctuelle

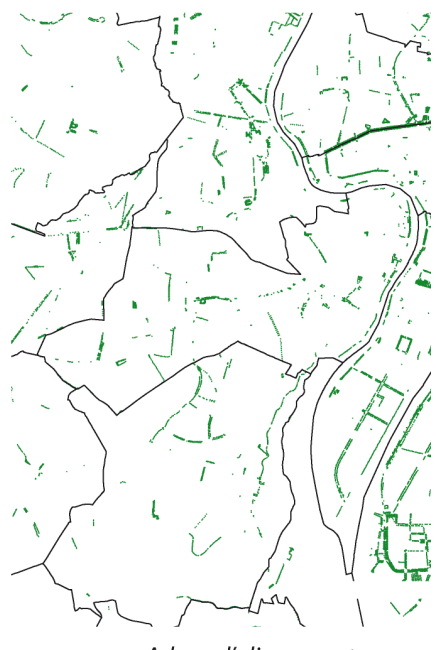

Arbres d'alignement

\section{passage sous forme maillée et établissement} des facteurs de quantification
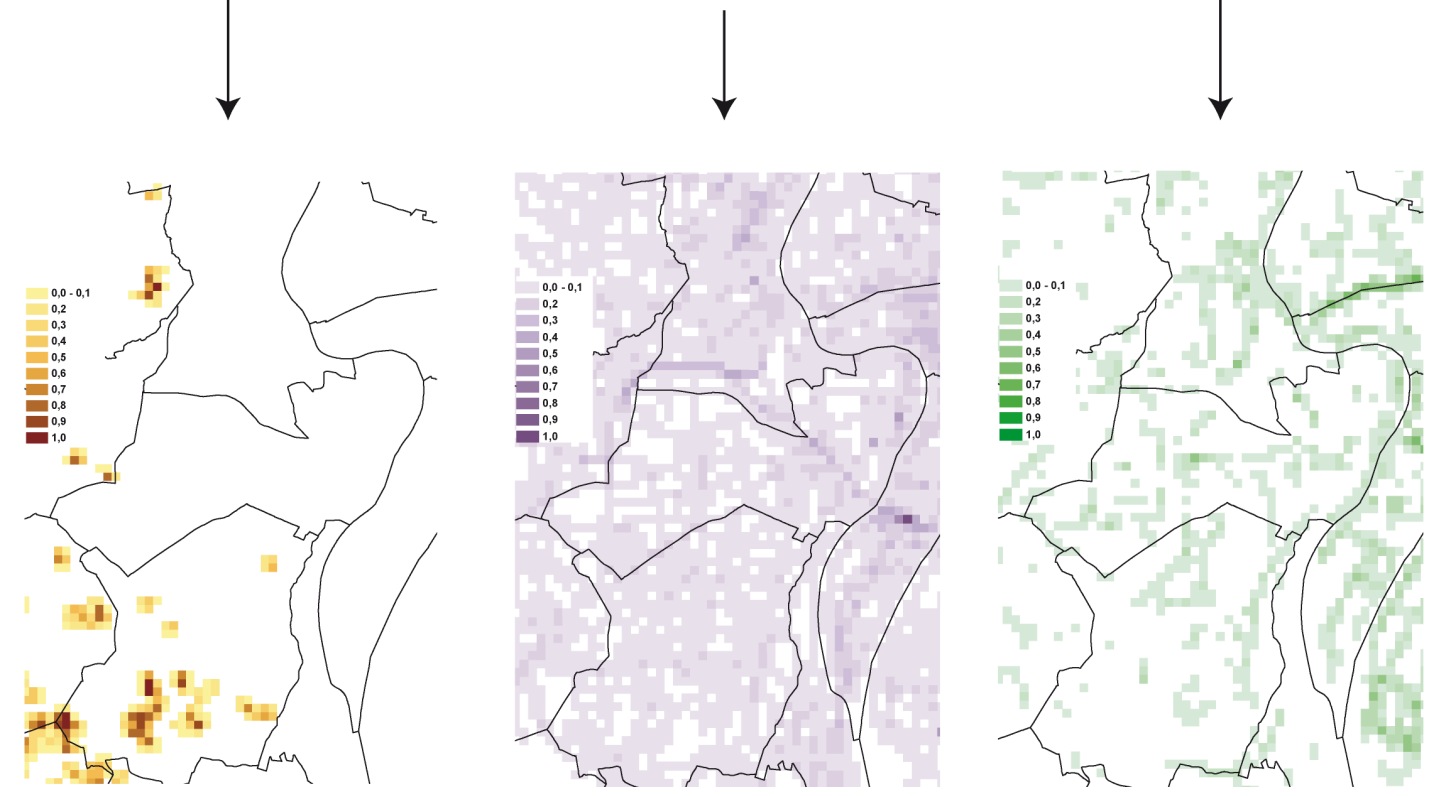

Figure 4. Exemple de conversion d'enjeux représentés respectivement sous forme surfacique, linéaire et ponctuelle à une forme maillée.

couplage doit respecter au mieux le zonage de la vulnérabilité territoriale, mais aussi celui de l'aléa, sans en modifier les contours initiaux, mais en fusionnant ces deux strates d'information. De cette manière, ce mode opératoire dépasse de ce fait la simple superposition des couches de vulnérabilité et d'aléa, avec l'utilisation respectives de hachures en surimpression d'aplats de couleurs, par exemple. Cela permet de visualiser le risque de manière directe, et non par la superposition d'informations, ce qui est souvent le cas. Ce croise- ment et la traduction sémiologique du risque peuvent se faire de diverses manières, en fonction de la traduction cartographique de la valeur d'aléa. En effet, sa "probabilité d'occurrence peut être estimée qualitativement (négligeable, faible, forte) ou quantitativement (pour les inondations par exemple, on définit des périodes de retour de 10,30 ou 100 ans, voire davantage)" (Veyret et Laganier, 2013).

Deux méthodes de représentation du risque sont proposées ici. La première repose sur le produit de la vulnérabilité terri- 
Tableau 3. Traductions quantificatives potentielles de l'appréciation qualitative d'un aléa.

\begin{tabular}{lrrr}
\hline & \multicolumn{3}{c}{ Aléa } \\
\hline $\begin{array}{l}\text { Appréciation } \\
\text { qualitative }\end{array}$ & faible & moyen & fort \\
\hline Traduction & & & \\
quantitative & 1 & 2 & 3 \\
& 1 & 5 & 10 \\
& 1 & 10 & 100 \\
& $?$ & $?$ & $?$
\end{tabular}

toriale par une note quantitative d'aléa. Cette pondération de l'aléa est en général une fonction linéaire de ses paramètres physiques, tels que son intensité, sa magnitude, sa hauteur d'eau, sa période de retour, etc. Cependant, l'aléa est traduit le plus souvent par des critères qualitatifs (par exemple: aléa fort, moyen et faible), et son accroissement n'est pas forcément linéaire, comme illustré dans le tableau 3. En effet, un aléa moyen est-il deux fois plus sévère qu'un aléa faible, ou l'est-il cinq fois, ou dix, etc. (tableau 3)? De ce fait, la traduction des critères qualitatifs de l'aléa en critères quantitatifs est critiquable (dans Granger, 2009; (Caradot et al., 2011, par exemple). En outre, il ne subsiste au final qu'une pondération du risque, et l'on ne sait si cette valeur est essentiellement due à celle de l'aléa ou à celle de la vulnérabilité des enjeux. En effet, "le calcul même du risque par multiplication de la probabilité d'occurrence d'un événement et de l'étendue du dommage potentiel est trompeur: d'après cette définition, le risque serait identique pour un événement rare à forte magnitude et pour un événement fréquent à faible magnitude" (Thomi, 2010). Par exemple, une note de risque de 10 peut aussi bien être le résultat d'un aléa de valeur 10 et d'une vulnérabilité de 1, que l'inverse (tableau 4).

La représentation spatiale du risque par le produit uniquement quantitatif de valeur d'aléa et de vulnérabilité apparaît donc insuffisante, étant donné la difficulté à retranscrire sous forme quantitative un aléa très souvent exprimé de manière qualitative, mais aussi car la note finale de risque ne permet plus de différencier la part respective de ces composantes. Ainsi, une sémiologie mixte, bidimensionnelle, fondée sur l'union des pondérations quantitatives de la vulnérabilité territoriale et les critères qualitatifs de l'aléa est aussi proposée, afin d'obtenir la vision du risque la plus précise possible (tableau 5). Cette légende en tableau à double entrée présente l'avantage de pouvoir se lire aussi bien horizontalement (vulnérabilité) que verticalement (aléa), en fonction de l'objectif de l'étude.
Tableau 4. Appréciation quantitative d'un risque par le produit de valeurs quantitatives d'aléa et de vulnérabilité (par exemple, on ne sait si la note finale de risque de 10 est issue d'une forte valeur d'aléa ou de vulnérabilité).

\begin{tabular}{lrrrr}
\hline & \multicolumn{4}{c}{ Valeurs d'aléa } \\
& 1 & 5 & 10 \\
\hline Valeurs de & & & & \\
vulnérabilité & 1 & 1 & 5 & 10 \\
& 5 & 5 & 25 & 50 \\
& 10 & 10 & 50 & 100 \\
\hline
\end{tabular}

\section{Résultats des fonctions de pondération et traitements cartographiques}

\subsection{Cartographie des vulnérabilités territoriales}

Les résultats obtenus permettent de prioriser la vulnérabilité des enjeux du Grand Lyon par l'établissement de fonctions de pondération. Elles regroupent tous les enjeux d'un même niveau de la décomposition hiérarchique en leur affectant un poids en fonction de leur vulnérabilité respective. Par exemple, pour la première ligne de la décomposition hiérarchique, la fonction de vulnérabilité globale est la suivante:

$$
\begin{aligned}
& \text { Vulnérabilitéglobale }=0,74 \times \mathrm{Vul}_{\text {humaine }}+0,06 \\
& \times \mathrm{Vul}_{\text {environnementale }}+0,20 \times \mathrm{Vul}_{\text {matérielle }}
\end{aligned}
$$

Cette fonction montre la part prépondérante des enjeux humains. Léone (2007) les qualifie même d'"enjeux suprêmes à préserver". Cependant, les parts des enjeux environnementaux et matériels ne sont pas équivalentes. En effet, d'après les experts, les enjeux matériels sont plus vulnérables à la submersion que les enjeux environnementaux. De la même manière, des fonctions de vulnérabilité sont obtenues pour chaque niveau de l'index. Par exemple, la fonction de vulnérabilité des réseaux est la suivante:

$$
\begin{aligned}
& \text { Vulnerabilité }_{\text {réseau }}=0.22 \times \mathrm{Vul}_{\text {eau potable }}+0.249 \\
& \times \mathrm{Vul}_{\text {assainissement }}+0.185 \times \mathrm{Vul}_{\text {électricité }}+0.085 \\
& \times \mathrm{Vul}_{\text {produits chimiques }}+0.104 \times \mathrm{Vul}_{\text {hydrocarbures }} \\
& +0.157 \times \mathrm{Vul}_{\text {télécommunications }}
\end{aligned}
$$

Le premier objectif de cette étude est la cartographie de la vulnérabilité des enjeux du Grand Lyon. La Fig. 5 représente spatialement, qualitativement et quantitativement la vulnérabilité humaine, environnementale et matérielle.

Le gradient décroissant centre - périphérie de vulnérabilité humaine est corrélé à la vulnérabilité matérielle (Fig. 5). En effet, les enjeux matériels, comme les bâtiments ou les infrastructures de transport, se regroupent dans le cœur urbain du Grand Lyon et se concentrent dans les secteurs de forte densité de population. Cette répartition des enjeux matériels dans l'agglomération explique la répartition de la vulnérabilité environnementale (Fig. 5). Ainsi, les secteurs pauvres 

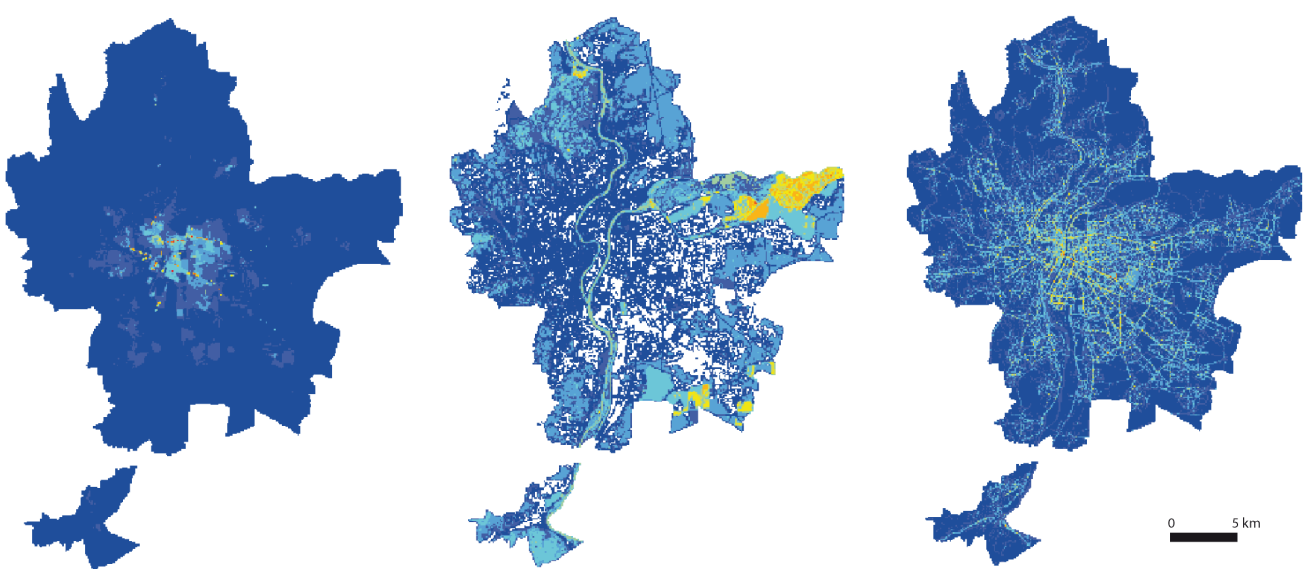

Faible vulnérabilité

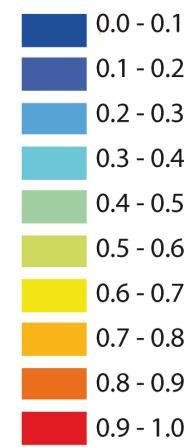

Forte vulnerabilité

Figure 5. Vulnérabilités humaine (à gauche), environnementale (au centre) et matérielle (à droite).

Tableau 5. Appréciation mixte d'un risque par le produit de valeurs qualitatives d'aléa et quantitatives de vulnérabilité (Ici et à la différence du tableau 3, la part respective des deux composantes du risque est préservée, pour une connaissance optimale du risque).

\begin{tabular}{lrrrr}
\hline & & \multicolumn{2}{c}{ Valeurs d'aléa } \\
& & faible (f) & moyen (m) & fort (F) \\
\hline Valeurs de & & & & \\
vulnérabilité & 1 & $1 \mathrm{f}$ & $5 \mathrm{~m}$ & $10 \mathrm{~F}$ \\
& 5 & $5 \mathrm{f}$ & $25 \mathrm{~m}$ & $50 \mathrm{~F}$ \\
& 10 & $10 \mathrm{f}$ & $50 \mathrm{~m}$ & $100 \mathrm{~F}$ \\
\hline
\end{tabular}

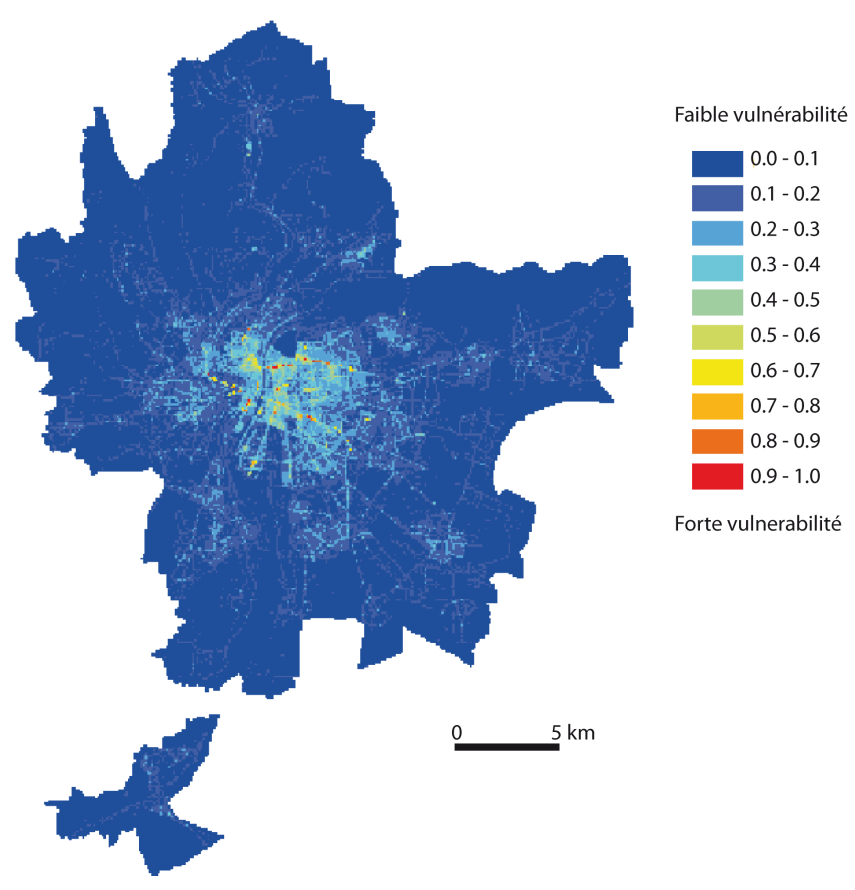

Figure 6. Vulnérabilité territoriale du Grand Lyon. en enjeux matériels sont dotés de forts enjeux environnementaux, et inversement. De ce fait, cette vulnérabilité environnementale se situe majoritairement en périphérie. De la même manière, la carte de vulnérabilité globale (Fig. 6) reste proche de celle de la vulnérabilité humaine, en raison du poids de la vulnérabilité humaine $(74 \%)$ dans la fonction de vulnérabilité globale.

\subsection{Cartographie des risques de submersion et de ruissellement}

La méthodologie de cartographie précédemment détaillée est appliquée sur le territoire du Grand Lyon aux risques de submersion (crues Rhône ou Saône), et au risque de ruissellement (Figs. 7 et 8), avec les deux types de légende proposées (quantitative et qualitative).

Ces cartographies permettent de mettre en évidence la forte nécessité de prise en compte de la variabilité spatiale de la vulnérabilité des enjeux. Ainsi, pour un aléa réparti sur l'ensemble du lit majeur du Rhône, la très forte disparité spatiale de la vulnérabilité conduit à une gestion de ce risque qui doit être adaptée très localement, comme nous l'indique la Fig. 7c, où l'on passe de la valeur $2 \mathrm{a}$ à la valeur $8 \mathrm{a}$, en un hectomètre seulement (sur deux mailles adjacentes).

Il est aussi très utile pour les décideurs d'avoir une vision précise de la vulnérabilité sur l'ensemble de l'agglomération lyonnaise, et non en quelques secteurs seulement, car la quasi-totalité du Grand Lyon peut potentiellement être affectée par des aléas d'origine pluviale. Ainsi, la Fig. 8 présente le risque de ruissellement sur l'agglomération lyonnaise. Nous constatons que des secteurs qui ne sont pas du tout concernés par l'aléa de submersion Rhône/Saône (Fig. 7) le sont fortement par l'aléa ruissellement (Fig. 8), notamment tout le nord-ouest de l'agglomération. 
a)

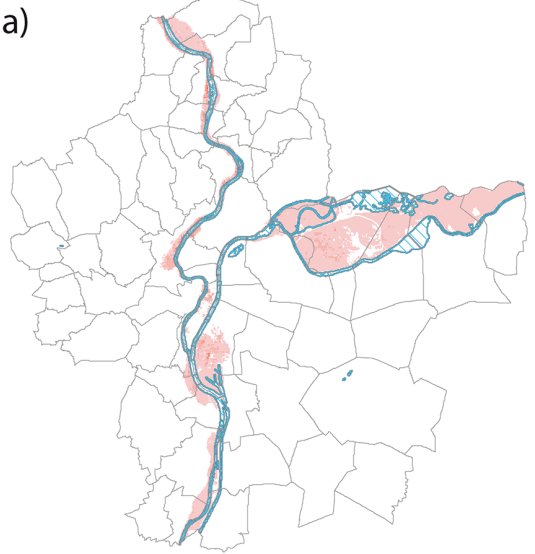

risque inondation

échelle quantitative

$[0 ; 1[$

$[1 ; 2[$

[2;3[

$[3 ; 4[$

$[4 ; 5[$

$[5 ; 6[$

$[6 ; 7[$

$[7 ; 8$

$[8 ; 9[$

$[9 ; 10]$

c)

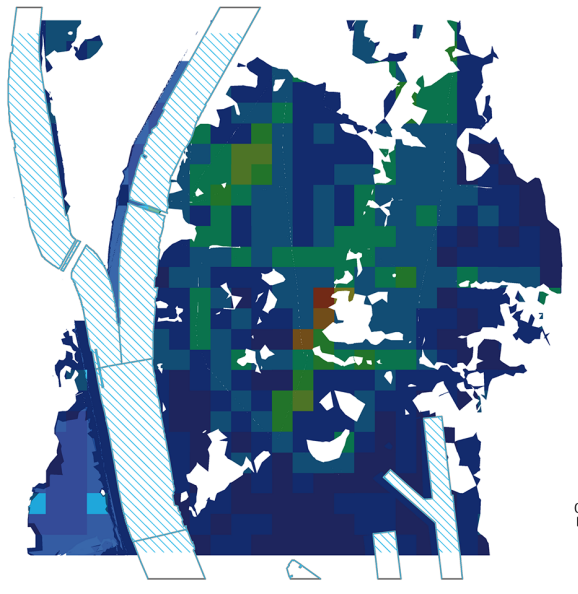

b)
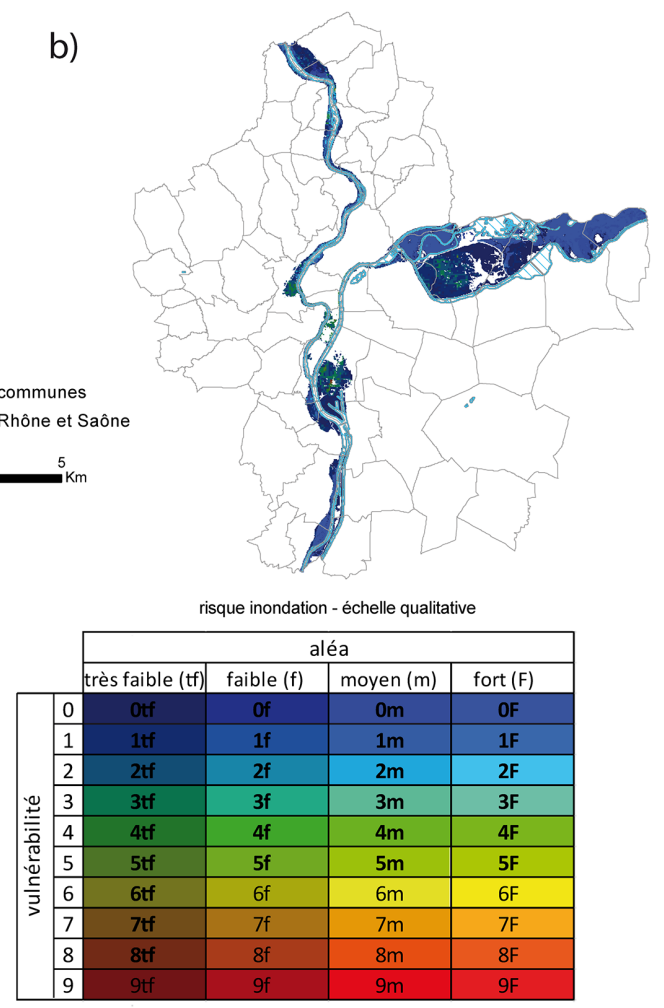

Figure 7. Risque de débordement du Rhône et de la Saône. (a) représentation quantitative; (b) représentation qualitative (association de l'indice de vulnérabilité et de l'intensité de l'aléa); (c) zoom de (b) sur le centre de Lyon en confluence du Rhône et de la Saône (seules les valeurs en gras dans la légende sont effectivement présentes dans cette vignette); (d) identique à (c) avec filtrage des valeurs de vulnérabilité inférieures à 3.

Enfin, il convient de remarquer la gestion efficace du risque de submersion car les zones à plus forte vulnérabilité sont uniquement exposées à un aléa très faible. En effet, la valeur de risque la plus haute n'est que de 9f (cf. Fig. 7). Il en est de même pour le risque de ruissellement, où les valeurs de risque les plus élevées sont de $5 \mathrm{~F}$ et de $7 \mathrm{f}$. Ces propositions méthodologiques de cartographies visent donc à pallier certaines faiblesses de travaux antérieurs, mais elles ne sont pas sans certaines limites, qu'il convient de prendre en compte.

\subsection{Applications et limites des représentations proposées}

La légende quantitative du risque, malgré les faiblesses préalablement énoncées, est plus facile à comprendre, de prime abord, que celle qualitative, qui associe directement les valeurs des deux composantes. En effet, pour le risque de submersion, la légende quantitative offre dix classes de valeurs, alors que la représentation qualitative en propose 40 . Cette 


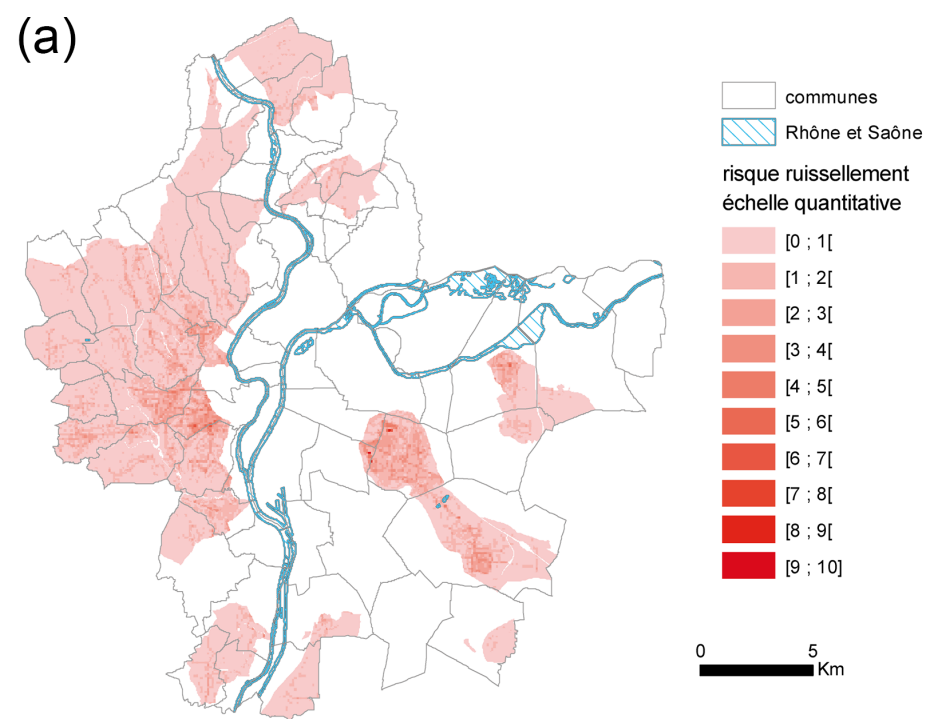

(b)
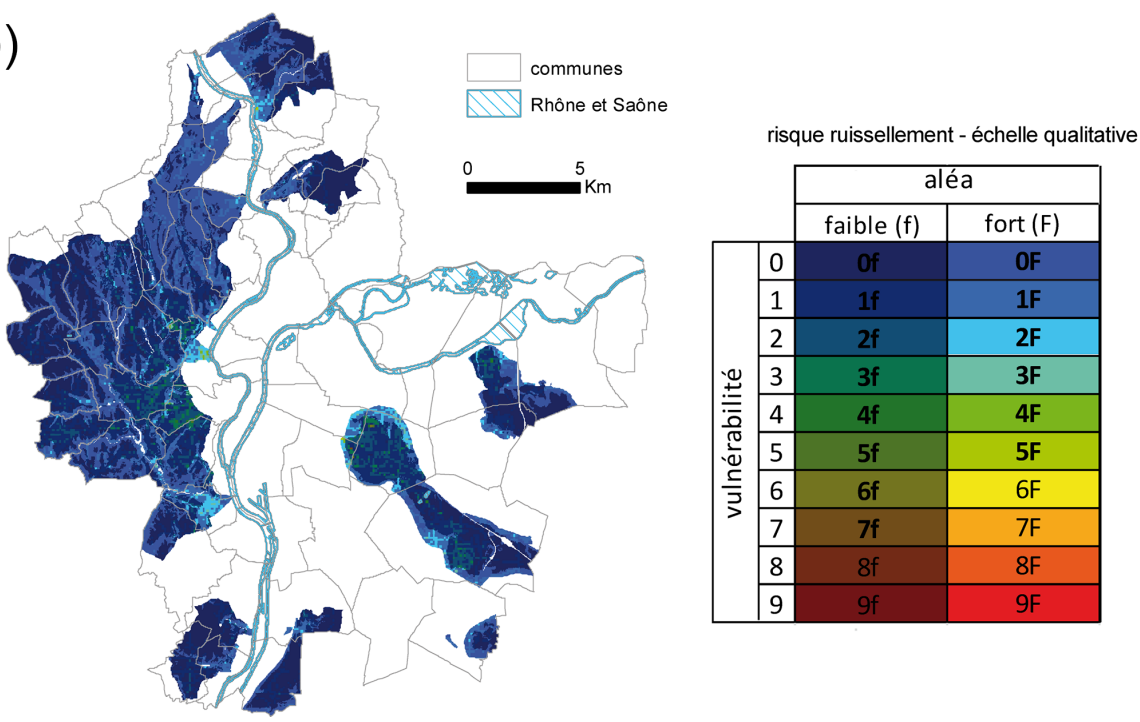

Figure 8. Risque de ruissellement. Représentation quantitative (a) et qualitative (b): association de l'indice de vulnérabilité et de l'intensité de l'aléa - seules les valeurs en gras sont effectivement présentes dans cette figure).

fine discrétisation de la valeur du risque constitue donc une faiblesse visuelle, mais est aussi un atout de précision. En outre, la différenciation visuelle de 40 teintes de couleurs différentes n'est pas aisée. La vulnérabilité est représentée en utilisant la couleur comme variable graphémique, et la valeur est utilisée pour distinguer les niveaux d'aléa (Bertin, 1975). Il convient de remarquer que l'inverse aurait aussi pu être utilisé, mais la couleur rouge la plus vive a été préférée pour la valeur de risque la plus élevée (9F). Cela en fait une sémiologie particulièrement adaptée à l'outil SIG, avec la possibilité de se focaliser sur une zone précise (cf. Fig. 6c et d), et d'étiqueter les valeurs pour une information détaillée. Le SIG offre en outre la possibilité d'afficher uniquement les valeurs fortes de vulnérabilité et d'aléa, et de filtrer les plus faibles (tel qu'en Fig. 6d, où les valeurs de vulnérabilité inférieures à 3 ont été filtrées). En outre, il convient de remarquer qu'en raison de l'universalité de la légende proposée, toutes les valeurs de risque potentielles ne se retrouvent pas forcément, car toutes les combinaisons de risque ne se produisent pas toujours sur le terrain (surtout pour les valeurs élevées, notamment lors d'une gestion efficace du risque). Par exemple, pour le risque de ruissellement, les valeurs $6 \mathrm{~F}, 7 \mathrm{~F}, 8 \mathrm{f}, 8 \mathrm{~F}, 9 \mathrm{f}$ et $9 \mathrm{~F}$ ne se retrouvent pas sur le Grand Lyon, et pour le risque de submersion, nous notons une absence des valeurs de $\mathrm{f}$ à $\mathrm{F}$ pour les aléas de 6 à 9 , et une absence complète de la dixième catégorie de risque (cf. Figs. 6 et 7). 


\section{Conclusion}

Pendant longtemps, la gestion du risque s'est uniquement appuyée sur la connaissance et la maîtrise de l'aléa. Les cartes de risque étaient souvent des zonages d'exposition à un aléa donné. La notion de vulnérabilité territoriale a fait son apparition plus récemment, et est maintenant partagée par de nombreuses disciplines scientifiques, ainsi que par les acteurs et les élus locaux. Elle apparaît aujourd'hui comme un champ de recherche à part entière. Les études visant à en améliorer sa connaissance ont montré leur utilité, mais aussi leurs limites. Nous avons donc proposé, dans un premier temps, une méthodologie dont le but est de qualifier et de quantifier spatialement cette vulnérabilité, adaptable à tous types d'aléas et en tous lieux, en tenant compte de la diversité des enjeux présents sur le territoire, et en recourant à des méthodes d'aide à la décision et à des traitements géomatiques par SIG. Ensuite, une cartographie globale du risque a été proposée, fondée sur les résultats de l'évaluation de la vulnérabilité territoriale et respectant les périmètres des zonages d'aléa. Cela a été possible par l'utilisation de procédés géomatiques novateurs, permettant le couplage de ces deux variables, et a permis de mettre en évidence la nécessité d'une gestion très localisée des risques en fonction de la vulnérabilité territoriale associée. En outre, deux types de légende ont été proposés (quantitative et qualitative), en fonction de la précision du niveau de risque désiré.

Les perspectives à ce travail sont nombreuses. En premier lieu, les facteurs de sensibilité des enjeux matériels et environnementaux seraient à prendre en compte. Ensuite, la mobilité des enjeux humains est à étudier, afin notamment de proposer des cartes de vulnérabilité humaine qui diffère en fonction du jour de la semaine (distinction jours ouvrés - week-end), ou en fonction du cycle nycthéméral (diurne - nocturne). In fine, cette méthodologie propose ainsi aux acteurs de gestion du risque un outil novateur de la visualisation spatiale du risque, qui nécessite cependant un certain apprentissage et mérite d'être manipulé avec précaution.

A German version of this article is available as a Supplement.

\section{The Supplement related to this article is available online at doi:10.5194/gh-70-333-2015-supplement.}

Remerciements. Les auteurs remercient vivement les relecteurs qui ont participé à l'amélioration de ce texte.

Edited by: O. Söderström

Reviewed by: two anonymous referees

\section{Références}

Agence d'urbanisme pour le développement de l'agglomération lyonnaise: Risques technologiques et vulnérabilités dans l'agglomération lyonnaise, Grand Lyon, Lyon, 48 pp., 2005.

Becouze-Lareure, C.: Multi-Level Approach of the Ecotoxicological Impact of a Combined Sewer Overflow on a Peri-Urban Stream, J. Water Resour. Prot., 04, 984-992, doi:10.4236/jwarp.2012.411114, 2012.

Bertin, J.: La Graphique et le traitement graphique de l'information, Flammarion, Paris, 192 pp., 1975.

Blaikie, P., Cannon, T., Davis, I., and Wisner, B.: At Risk: Natural Hazards, People, Vulnerability, and Disasters, Routledge, London, UK, 124 pp., 1994.

Blancher, P., Paquiet, P., et Zampa, C.: Industries chimiques et territoire: contraintes et opportunités de développement/Chemical industries and their spatial setting: constraints and opportunities for development, Rev. Géographie Lyon, 71, 23-30, 1996.

Bourrelier, P.-H.: Introduction du no 43 de Responsabilité et environnement, Responsab. Environ., 2, 7-9, 2006.

Braud, I., Breil, P., Thollet, F., Lagouy, M., Branger, F., Jacqueminet, C., Kermadi, S., and Michel, K.: Evidence of the impact of urbanization on the hydrological regime of a mediumsized periurban catchment in France, J. Hydrol., 485, 5-23, doi:10.1016/j.jhydrol.2012.04.049, 2013.

Caradot, N., Granger, D., Chapgier, J., Cherqui, F., and Chocat, B.: Urban flood risk assessment using sewer flooding databases, Water Sci. Technol., 64, 832, doi:10.2166/wst.2011.611, 2011.

Cherqui, F.: Méthodologie d'évaluation d'un projet d'aménagement durable d'un quartier. Méthode Adequa, thèse, Université de La Rochelle, La Rochelle, 202 pp., 2005.

Chocat, B.: Encyclopédie de l'hydrologie urbaine et de l'assainissement, Tech \& Doc., Lavoisier, Paris, 1124 pp., 1997.

Combe, C.: La ville endormie? Le risque d'inondation à Lyon. Approche géohistorique et systémique du risque de crue en milieu urbain et périurbain, thèse, Université Lumière Lyon, 2, 467 pp., 2007.

Dauphiné, A. et Provitolo, D.: Risques et catastrophes: observer, spatialiser, comprendre, gérer, Armand Colin, Paris, 416 pp., 2013.

Demoraes, F. et D’Ercole, R.: Risques et accessibilité des lieux dans le District Métropolitain de Quito (Équateur), Mappemonde, (95(3-2009)), 1-20, 2009.

D' Ercole, R. et Pigeon, P.: L'expertise internationale des risques dits naturels: intérêt géographique/Geographical relevance of natural risk assessment on an international scale, Ann. Géographie, 108, 339-357, doi:10.3406/geo.1999.21777, 1999.

Fedeski, M. and Gwilliam, J.: Urban sustainability in the presence of flood and geological hazards: The development of a GIS-based vulnerability and risk assessment methodology, Landsc. Urban Plan., 83, 50-61, doi:10.1016/j.landurbplan.2007.05.012, 2007.

Fuchs, S., Birkmann, J., and Glade, T.: Vulnerability assessment in natural hazard and risk analysis: current approaches and future challenges, Nat. Hazards, 64, 1969-1975, doi:10.1007/s11069012-0352-9, 2012.

Füssel, H.-M. and Klein, R. J. T.: Climate Change Vulnerability Assessments: An Evolution of Conceptual Thinking, Clim. Change, 75, 301-329, doi:10.1007/s10584-006-0329-3, 2006. 
Gaume, E., Bain, V., Bernardara, P., Newinger, O., Barbuc, M., Bateman, A., Blaškovičová, L., Blöschl, G., Borga, M., Dumitrescu, A., Daliakopoulos, I., Garcia, J., Irimescu, A., Kohnova, S., Koutroulis, A., Marchi, L., Matreata, S., Medina, V., Preciso, E., Sempere-Torres, D., Stancalie, G., Szolgay, J., Tsanis, I., Velasco, D., and Viglione, A.: A compilation of data on European flash floods, J. Hydrol., 367, 70-78, doi:10.1016/j.jhydrol.2008.12.028, 2009.

Gilbert, C.: L'expertise et la science, in atelier RDT, Paris, 61-63, 2006.

Gleyze, J. F. et Reghezza, M.: La vulnérabilité structurelle comme outil de compréhension des mécanismes d'endommagement, Géocarrefour Rev. Géographie Lyon, 82, 17-26, 2007.

Granger, D.: Méthodologie d'aide à la gestion durable des eaux urbaines, thèse, INSA de Lyon, Lyon, 210 pp., 2009.

Griot, C.: Vulnérabilité et risques liés au transport de matières dangereuses: évaluation en vue de la préparation aux interventions de la Sécurité Civile, thèse, Montpellier 3, Montpellier, 584 pp., 2003.

Griot, C.: Des territoires vulnérables face à un risque majeur: le transport de matières dangereuses. Proposition d'un outil d'aide à la gestion de crise, Géocarrefour, 82, 51-63, 2007.

Griot, C. et Ayral, P.-A.: Terminologie en sciences du risque, p. 87, Mèzes, France, 2001.

Haziza, E.: Contribution au développement de stratégies préventives face au risque de crues éclairs, thèse, Ecole des Mines de Paris, Paris, 409 pp., 2007.

IPCC: Summary for policymakers, in: Climate Change 2014: Impacts, Adaptation, and Vulnerability. Part A: Global and Sectoral Aspects. Contribution of Working Group II to the Fifth Assessment Report of the Intergovernmental Panel on Climate Change, edited by: Field, C. B., Barros, V. R., Dokken, D. J., Mach, K. J., Mastrandrea, M. D., Bilir, T. E., Chatterjee, M., Ebi, K. L., Estrada, Y. O., Genova, R. C., Girma, B., Kissel, E. S., Levy, A. N., MacCracken, S., Mastrandrea, P. R., and White, L. L., Cambridge University Press, Cambridge, United Kingdom and New York, NY, USA, 1-32, 2014.

Jongman, B., Koks, E. E., Husby, T. G., and Ward, P. J.: Increasing flood exposure in the Netherlands: implications for risk financing, Nat. Hazards Earth Syst. Sci., 14, 1245-1255, doi:10.5194/nhess-14-1245-2014, 2014.

Kappes, M. S., Papathoma-Köhle, M., and Keiler, M.: Assessing physical vulnerability for multi-hazards using an indicator-based methodology, Appl. Geogr., 32, 577-590, doi:10.1016/j.apgeog.2011.07.002, 2012.

Kaźmierczak, A. and Cavan, G.: Surface water flooding risk to urban communities: Analysis of vulnerability, hazard and exposure, Landsc. Urban Plan., 103, 185-197, doi:10.1016/j.landurbplan.2011.07.008, 2011.

Kendrick, J. D. and Saaty, D.: Use analytic hierarchy process for project selection, ASQ Six Sigma Forum Magazine, 6, 22-29, 2007.

Kienberger, S., Lang, S., and Zeil, P.: Spatial vulnerability units expert-based spatial modelling of socio-economic vulnerability in the Salzach catchment, Austria, Nat. Hazards Earth Syst. Sci., 9, 767-778, doi:10.5194/nhess-9-767-2009, 2009.

Koks, E. E., Jongman, B., Husby, T. G., and Botzen, W. J. W.: Combining hazard, exposure and social vulnerability to provide les- sons for flood risk management, Environ. Sci. Policy, 47, 42-52, doi:10.1016/j.envsci.2014.10.013, 2015.

Kouyi, G. L., Bret, P., Didier, J.-M., Chocat, B., and Billat, C.: The use of CFD modelling to optimise measurement of overflow rates in a downstream-controlled dual-overflow structure, Water Sci. Technol., 64, 521, doi:10.2166/wst.2011.162, 2011.

Kreimer, A., Arnold, M., and Carlin, A.: Building safer cities: the future of disaster risk, World Bank Publications, Washington, DC, 324 pp., ISBN0-8213-5497-3, 2003.

Kron, W.: Flood risk $=$ hazard $\times$ exposure $\times$ vulnerability, Keynote Lecture, Proceedings of the Flood Defence, 17 pp., 2002.

Léone, F.: Caractérisation des vulnérabilités aux « catastrophes naturelles $\gg$ : contribution à une évaluation géographique multirisque, HDR, université de Montpellier III, Montpellier, 2007.

Lepioufle, J.-M., Leblois, E., and Creutin, J.-D.: Variography of rainfall accumulation in presence of advection, J. Hydrol., 464 465, 494-504, doi:10.1016/j.jhydrol.2012.07.041, 2012.

Llasat, M. C., Llasat-Botija, M., Prat, M. A., Porcú, F., Price, C., Mugnai, A., Lagouvardos, K., Kotroni, V., Katsanos, D., Michaelides, S., Yair, Y., Savvidou, K., and Nicolaides, K.: High-impact floods and flash floods in Mediterranean countries: the FLASH preliminary database, Adv. Geosci., 23, 47-55, doi:10.5194/adgeo-23-47-2010, 2010.

Lummen, N. S. and Yamada, F.: Implementation of an integrated vulnerability and risk assessment model, Nat. Hazards, 73, 10851117, doi:10.1007/s11069-014-1123-6, 2014.

Marchi, L., Borga, M., Preciso, E., and Gaume, E.: Characterisation of selected extreme flash floods in Europe and implications for flood risk management, J. Hydrol., 394, 118-133, doi:10.1016/j.jhydrol.2010.07.017, 2010.

Marzo, E., Busini, V., and Rota, R.: Definition of a short-cut methodology for assessing the vulnerability of a territory in naturaltechnological risk estimation, Reliab. Eng. Syst. Saf., 134, 92 97, doi:10.1016/j.ress.2014.07.026, 2015.

Meschinet de Richemond, N. et Reghezza, M.: La gestion du risque en France: contre ou avec le territoire?, Ann. Géographie, 673, 248-267, doi:10.3917/ag.673.0248, 2010.

Messner, F. and Meyer, V.: Flood damage, vulnerability and risk perception-challenges for flood damage research, NATO Science Series, Springer, the Netherlands, 149-167, 2006.

Moser, S. C.: Now more than ever: the need for more societally relevant research on vulnerability and adaptation to climate change, Appl. Geogr., 30, 464-474, 2010.

November, V.: Les territoires du risque: le risque comme objet de réflexion géographique, edited by: Lang, P., Berne, 332 pp., Suisse, 2002.

O'Brien, K., Eriksen, S., Nygaard, L. P., and Schjolden, A.: Why different interpretations of vulnerability matter in climate change discourses, Clim. Policy, 7, 73-88, doi:10.1080/14693062.2007.9685639, 2007.

Peduzzi, P., Dao, H., Herold, C., and Mouton, F.: Assessing global exposure and vulnerability towards natural hazards: the Disaster Risk Index, Nat. Hazards Earth Syst. Sci., 9, 1149-1159, doi:10.5194/nhess-9-1149-2009, 2009.

Penning-Rowsell, E., Floyd, P., Ramsbottom, D., and Surendran, S.: Estimating injury and loss of life in floods: a deterministic framework, Nat. Hazards, 36, 43-64, 2005.

Poussin, J. K., Bubeck, P., Aerts, J. C. J. H., and Ward, P. J.: Potential of semi-structural and non-structural adaptation strate- 
gies to reduce future flood risk: case study for the Meuse, Nat. Hazards Earth Syst. Sci., 12, 3455-3471, doi:10.5194/nhess-123455-2012, 2012.

Propeck-Zimmermann, E., Saint-Gérand, T., et Bonnet, E.: Nouvelles approches ergonomiques de la cartographie des risques industriels, Mappemonde, 4, 19, 2009.

Reghezza, M.: Géographes et gestionnaires face à la vulnérabilité métropolitaine. Quelques réflexions autour du cas francilien, Ann. Géographie, 669, 459-477, doi:10.3917/ag.669.0459, 2009.

Renard, F.: Le risque pluvial en milieu urbain. De la caractérisation de l'aléa à l'évaluation de la vulnérabilité: le cas du Grand Lyon, thèse, Université Jean Moulin Lyon 3, Lyon, 528 pp., 2010.

Renard, F. et Chapon, P.-M.: Une méthode d'évaluation de la vulnérabilité urbaine appliquée à l'agglomération lyonnaise, L'Espace Géographique, 39, 35-50, 2010.

Rufat, S.: L'estimation de la vulnérabilité urbaine, un outil pour la gestion du risque, Géocarrefour, 82, 7-16, doi:10.4000/geocarrefour.1397, 2007.

Ruin, I. et Lutoff, C.: Vulnérabilité face aux crues rapides et mobilités des populations en temps de crise, Houille Blanche, 6, 114119, doi:10.1051/lhb:200406016, 2004.

Saaty, T. L.: The Analytic Hierarchy Process, Mc Graw-Hill, NewYork, 287 pp., 1980.

Saaty, T. L.: Highlights and critical points in the theory and application of the analytic hierarchy process, Eur. J. Oper. Res., 74, 426-447, 1994.
Shi, W. and Zeng, W.: Genetic k-Means Clustering Approach for Mapping Human Vulnerability to Chemical Hazards in the Industrialized City: A Case Study of Shanghai, China, Int. J. Environ. Res. Public. Health, 10, 2578-2595, doi:10.3390/ijerph10062578, 2013.

Tarolli, P., Borga, M., Morin, E., and Delrieu, G.: Analysis of flash flood regimes in the North-Western and South-Eastern Mediterranean regions, Nat. Hazards Earth Syst. Sci., 12, 1255-1265, doi:10.5194/nhess-12-1255-2012, 2012.

Thomi, L.: Rôle des paramètres sociopolitiques et des connaissances dans la gestion des risques hydrologiques, thèse, Université de Lausanne, Lausanne, 538 pp., 2010.

Tixier, J., Dandrieux, A., Dusserre, G., Bubbico, R., Mazzarotta, B., Silvetti, B., Hubert, E., Rodrigues, N. and Salvi, O.: Environmental vulnerability assessment in the vicinity of an industrial site in the frame of ARAMIS European project, J. Hazard. Mater., 130, 251-264, 2006.

UNISDR: Global Assessment Report on Disaster Risk Reduction. Revealing Risk, Redefining Development, United Nations International Strategy for Disaster Reduction Secretariat, Geneva, Switzerland, 128 pp., 2011.

Veyret, Y. et Laganier, R.: Atlas des risques en France. Prévenir les catastrophes naturelles et technologiques, Autrement, 96 pp., 2013.

Veyret, Y. et Reghezza, M.: Vulnérabilité et risques. L'approche récente de la vulnérabilité, Ann. Mines, 7, 9-14 2006. 